\title{
Incidencia y factores de riesgo de la fractura de fémur proximal por osteoporosis
}

\author{
María Teresa Mosquera, ${ }^{1}$ Daniel Luis Maurel, ${ }^{2}$ Salvador Pavón, ${ }^{3}$ \\ Agustín Arregui, ${ }^{1}$ Carlos Moreno ${ }^{3}$ y Jesús Vázquez ${ }^{1}$
}

RESUMEN Todos los años se producen en el mundo más de un millón de fracturas de fémur proximal, sobre todo en personas de edad avanzada. Dado el continuo envejecimiento de las poblaciones, las fracturas aumentarán año tras año y constituirán un problema cada vez más grave de salud pública. Se espera que el mayor aumento de dichas fracturas ocurra en América Latina alrededor del 2050. Teniendo en cuenta que cerca de $70 \%$ de las fracturas atraumáticas en personas mayores de 45 años de edad se deben a osteoporosis, se diseñó un estudio de casos y controles en la ciudad de Mar del Plata, Argentina, para conocer la incidencia de fracturas de fémur proximal por osteoporosis y los factores de riesgo asociados. Entre el 1 de agosto de 1992 y el 31 de julio de 1993 se registraron todos los casos de fracturas de fémur proximal por osteoporosis en personas mayores de 50 años de edad que acudieron a cualquiera de los 30 centros de salud públicos y privados de la ciudad. Se registró un total de 246 casos. La tasa de incidencia por 100000 habitantes en la población mayor de 50 años fue de 259 en mujeres y de 92 en varones, con una relación de 2,8:1. La incidencia fue siempre mayor a mayor edad y sobre todo a partir de los 75 años. Los factores asociados con aumento del riesgo de fractura de fémur proximal con significación estadística fueron: antecedentes de enfermedades neurológicas, consumo de psicofármacos, consumo de alcohol, fracturas previas, enfermedades cardiovasculares y menor consumo de lácteos. No se observaron diferencias entre los casos y los controles con respecto a edad de inicio de la menopausia, peso, talla, actividad previa, hábito de fumar o exposición al sol, como así tampoco en el porcentaje de mujeres que habían tenido ooforectomías.

Se considera que $70 \%$ de las fracturas óseas atraumáticas en personas mayores de 45 años se deben a osteoporosis. En 1990 ocurrieron aproximadamente 1,7 millones de fracturas de

\footnotetext{
1 Hospital Interzonal General de Agudos, Sección de Metabolismo Fosfocálcico, Mar del Plata, Argentina.

2 Instituto Nacional de Epidemiología Dr. Juan H. Jara, Mar del Plata, Argentina. La correspondencia y pedidos de separatas deben enviarse a D.L. Maurel, a la siguiente dirección: Instituto Nacional de Epidemiología Dr. Juan H. Jara, Ituzaingó 3520, Mar del Plata 7600, Argentina.

3 Hospital Privado de Comunidad., Servicio de Ortopedia y Traumatología, Mar del Plata, Argentina.
}

fémur proximal en todo el mundo (1). Actualmente hay más de 325 millones de personas mayores de 65 años en el planeta, cifra que, según se estima, incrementará a unos 1500 millones para el 2050. Puesto que la osteoporosis es un proceso patológico continuo en el cual el aumento de la incidencia es exponencial en relación a la edad, en ese año Asia, que tendrá el mayor número de ancianos, llegará a tener también el mayor número de fracturas de fémur proximal de todo el mundo, las cuales se incrementarán de menos de un millón a más de cuatro millones $(1,2)$. No obstante, según las estima- ciones estadísticas, el mayor porcentaje de aumento ocurrirá en América Latina. Para poder afrontar este problema de salud pública, es preciso establecer criterios de prevención.

El costo de la fractura de cadera en los Estados Unidos de América es, actualmente, de \$US 10020 millones al año $(3,4)$. La morbilidad y mortalidad debidas a esas fracturas (5) redundan en una reducción de la esperanza de vida de $12 \%$ o más, pues la mortalidad es mayor en los 3 a 4 meses siguientes (6). La mortalidad posfractura es de 3 a $4 \%$ a los 50 años de edad y de 28 a $30 \%$ a los 80 años. Después de sufrir una 
fractura de cadera, aproximadamente $50 \%$ no pueden caminar y más de $50 \%$ son incapaces de vivir independientemente (5-18).

A pesar de que la pérdida ósea por envejecimiento es universal y de que todas las personas pierden constantemente hueso cortical y trabecular a medida que envejecen $(20,21)$, solamente un porcentaje sufre fracturas (19). Aproximadamente $25 \%$ de las mujeres posmenopáusicas desarrollan osteoporosis y una tercera parte de las mujeres mayores de 65 años sufren fracturas vertebrales $(22,23)$. A partir de los 50 años, la mujer de raza blanca corre un riesgo de sufrir una fractura de la cadera de 15,6 a $17,5 \%$ y el hombre de raza blanca, un riesgo de 5,2 a $6,0 \%$. El riesgo es de 5,6\% para la mujer de raza negra y de $2,8 \%$ para el hombre de raza negra $(24,25)$. A los 90 años, aproximadamente un tercio de las mujeres han sufrido fractura de cadera, frente a solo de 15 a $20 \%$ de los hombres. Los pacientes con fractura de fémur proximal tienen de 3 a 10 veces mayor frecuencia de fracturas vertebrales, pero hay una menor correlación con la fractura de Colles (26).

Sin embargo, la menor masa ósea no parece ser la única causa determinante de las fracturas en ancianos; hay otros factores implicados (26-28). Esto significa que no solo debemos tener en cuenta la reducción cuantitativa de la masa ósea, sino además otros factores cualitativos, como la inadecuada reparación de las microfracturas causadas por fatiga, arquitectura anormal u osteomalacia (29-31). También se ha determinado que la resistencia ósea disminuye con la edad en ambos sexos $(32,33)$ y que en ello además ejerce influencia el medio; por ejemplo, los japoneses nacidos en Hawai tienen una mayor masa ósea que los nacidos en el Japón, aun con iguales ascendientes (34).

El objetivo de este trabajo fue conocer la incidencia de la fractura de fémur proximal por osteoporosis en Mar del Plata, Argentina, en personas de ambos sexos mayores de 49 años, ya que esta es la más importante de las fracturas por osteoporosis debido al impacto que tiene en la salud pública en función de la morbilidad, la mortalidad y los costos. Además, se procuró determinar cuáles eran algunos de los factores de riesgo más importantes relacionados con la osteoporosis y ese tipo de fractura.

\section{MATERIALES Y MÉTODOS}

Se realizó un estudio de casos y controles, que comenzó con el registro de todos los pacientes que tuvieron fracturas de fémur proximal durante el período comprendido entre agosto de 1992 y julio de 1993 y que fueron atendidos por esa causa en cualquiera de los 30 centros de salud públicos y privados de Mar del Plata. Se realizó un trabajo epidemiológico prospectivo, registrando todos los casos nuevos que ocurrían en la ciudad. Los pacientes y controles de ambos sexos se seleccionaron de acuerdo con dos criterios: ser mayores de 50 años de edad y tener 1 año o más de residencia en Mar del Plata. Las cifras poblacionales se obtuvieron del censo nacional de 1991, en el que se registró una población total de Mar del Plata de 541277 habitantes y 134718 personas mayores de 50 años, de las cuales 72804 eran mujeres y 61914 eran hombres.

En el estudio se incluyeron los casos de fractura del extremo proximal del fémur con diagnóstico radiológico confirmado, que ocurrieron como consecuencia de un esfuerzo habitual, caídas u otros traumatismos leves o moderados. Las fracturas del extremo proximal de fémur se dividieron en dos grupos: a) extracapsulares (intertrocantéreas y subtrocantéreas) y b) intracapsulares (subcapitales y cervicales). No se estudiaron las fracturas por otras causas, ya que no se consideraban consecuencia de una menor resistencia ósea.

Se excluyeron del estudio de factores de riesgo las fracturas por accidentes graves o por cáncer, así como las de pacientes con alteraciones mentales que restaran confiabilidad a la información impartida. Se seleccionaron como controles hombres y mujeres mayores de 49 años, con 1 año o más de residencia en la ciudad, que concu- rrían solos a los centros de salud para controles clínicos o acompañados a los servicios de clínica médica, traumatología o metabolismo fosfocálcico y que aceptaron voluntariamente someterse a la misma evaluación que los casos registrados. Los casos y los controles se eligieron simultáneamente y se aparearon por sexo y edad. La información personal fue recogida por dos médicos traumatólogos que entrevistaron a casos y controles en los centros asistenciales.

La evaluación de factores de riesgo se basó en las siguientes variables:

1. Funcionamiento de las extremidades inferiores antes de la fractura, en particular la necesidad de asistencia en las actividades de la vida diaria en el hogar. Se interrogó a los sujetos sobre la ayuda que requerían para llevar a cabo cuatro actividades: caminar de un extremo a otro de una habitación; ponerse de pie; caminar afuera y subir escaleras. Por cada dificultad indicada se les adjudicó un punto. A los sujetos que estaban inmovilizados en silla de ruedas o cama se les adjudicó un valor de 5 .

2. Trastornos visuales: se les interrogó sobre la presencia o no de cataratas o glaucoma.

3. Mecanismo del trauma que causó la fractura: si estaba acostado, sentado o de pie cuando ocurrió.

4. Enfermedades concomitantes o previas a la fractura (neurológicas, metabólicas, cardiovasculares) y otras fracturas.

5. Ingesta de medicamentos previa a la fractura (corticosteroides, estrógenos, vitaminas, tiazidas, psicofármacos, suplementos de calcio y otros.

6. Ingesta regular de productos lácteos (leche o equivalentes, en cantidad de vasos al día).

7. Ingesta regular cuantitativa de alcohol, en cantidad de vasos a la semana.

8. Tabaquismo (hábito de fumar).

9. Exposición al sol (horas a la semana).

10. Antecedentes familiares de osteoporosis. 
11. Antecedentes personales de ooforectomía.

12. Historia reproductiva: se dividió al grupo de mujeres en nulíparas y no nulíparas, por grado de paridad y por edad al inicio de la menopausia. La menopausia se definió como temprana antes de los 40 años y tardía después de los 51.

13. Parámetros corporales: índice de Quetelet o índice de masa corporal (IMC) e índice cintura/cadera (ICC). El ICC se calcula midiendo el diámetro menor de la cintura y el máximo de la cadera. También se midieron la talla y el peso.

14. Presión arterial diastólica y sistólica, y frecuencia cardíaca.

15. Localización de la fractura de fémur (derecha o izquierda, extracapsular o intracapsular).

16. Trastornos y problemas de los pies: se registró la presencia o no de trastornos de apoyo, Hallus valgus y otros.

17. Estado de la dentadura: se registró la pérdida de piezas dentarias.

18. Ingesta de calcio. Esta se calculó de forma semicuantitativa, preguntando sobre el consumo de leche, queso, yogur y crema, y dividiéndolo en tres clasificaciones: consumo nulo, 0; de 1 a 2 vasos al día, I; y 3 o más vasos al día, II.

Los datos se codificaron, tabularon y analizaron con el programa computadorizado Epi Info. En el análisis de resultados se utilizaron diversas medidas estadísticas: media, desviación estándar, razón de posibilidades (odds ratio) y ji cuadrado $\left(\chi^{2}\right)$. Se utilizó la razón de posibilidades como aproximación al riesgo relativo. Para los factores de riesgo se realizó un análisis bivariado.

\section{RESULTADOS}

\section{Incidencia de fractura proximal de fémur}

El número de fracturas registradas en ambos sexos durante los 12 meses del estudio ascendió a 246. De ellas, 203 (83\%) ocurrieron en pacientes mayores de 70 años de edad y 43 (17\%), en personas de 50 a 69 años de edad. La incidencia específica de la fractura proximal de fémur según sexo y edad fue mayor en las mujeres y mostró un aumento exponencial con la edad.

La incidencia general en el período de estudio fue de 182,6 por 100000 habitantes al año; la de varones, 92,1 y la de mujeres, 259,6. Desagregada por grupos de edad, la incidencia en mujeres fue como sigue: 50 a 59 años de edad, 18,5; 60 a 69 años, 94,0; y 70 años o más, 946,2. En los varones se encontraron los siguientes valores: 50 a 59 años de edad, 3,4; 60 a 69 años, 70,5; y 70 años o más, 326,6. La relación entre las fracturas extracapsulares y las intracapsulares fue equivalente en ambos sexos y en todos los grupos de edad. De las 246 fracturas de fémur proximal registradas, 189 (76,8\%) ocurrieron en mujeres y $57(23,2 \%)$ en hombres, con una razón de mujer a varón de 2,82:1.

\section{Factores de riesgo}

Las fracturas registradas en hombres y mujeres mensualmente no muestran variaciones relacionadas con los cambios estacionales. Respecto a actividad física previa, 80,6\% de los individuos que sufrieron fracturas caminaban sin asistencia antes de que esta ocurriera, pero el resto caminaba con ayuda.

La gran mayoría de los sujetos (95\%) estaban de pie cuando sobrevino el incidente que resultó en la fractura de fémur. En cuanto al mecanismo del trauma, en $94,4 \%$ de los casos la caída se produjo desde la posición de pie; en $49,9 \%$, la fractura ocurrió en el lado izquierdo y en 50,1\%, en el lado derecho (cuadro 1 y figura 1 ).

Como indica el cuadro 1, al comparar a los pacientes con fractura proximal de fémur y los controles, no se observaron diferencias significativas respecto a peso ni IMC, si bien en los casos con IMC elevado, el porcentaje de fracturas fue menor. Más de la mitad $(56 \%)$ de los pacientes tenían IMC menores de 25. Por lo que se refiere al ICC, casi toda la población estudiada se encontraba dentro del recorrido de 0,8 a 1,0, sin diferencias significativas entre casos y controles.

Se observaron diferencias importantes en las cifras de presión arterial sistólica. Los casos presentaron valores más elevados con diferencias estadísticamente significativas $(P<0,02)$. De los pacientes con fracturas, 59\% tenían presión arterial sistólica mayor de 150 mmHg frente a $39 \%$ de los controles. Los valores de 140 a 149 correspondieron a $25 \%$ de los casos y $35 \%$ de los

CUADRO 1. Incidencia de fractura de fémur proximal, según grupo de edad y sexo. Mar del Plata, Argentina, 1992-1993

\begin{tabular}{|c|c|c|c|c|c|c|c|c|}
\hline \multirow[b]{3}{*}{ Edad (años) } & \multicolumn{4}{|c|}{ Mujeres } & \multicolumn{4}{|c|}{ Hombres } \\
\hline & \multirow[b]{2}{*}{ Población } & \multicolumn{2}{|c|}{ Fracturas } & \multirow{2}{*}{$\begin{array}{c}\text { Tasa } \\
\left(\text { por } 10^{5}\right)\end{array}$} & \multirow[b]{2}{*}{ Población } & \multicolumn{2}{|c|}{ Fracturas } & \multirow{2}{*}{$\begin{array}{c}\text { Tasa } \\
\left(\text { por } 10^{5}\right)\end{array}$} \\
\hline & & No. & $\%$ & & & No. & $\%$ & \\
\hline $50-59$ & 32376 & 6 & 3,2 & 18,5 & 29186 & 1 & 1,8 & 3,4 \\
\hline 60-69 & 23412 & 22 & 11,6 & 94,0 & 19868 & 14 & 24,6 & 70,5 \\
\hline $70+$ & 17016 & 161 & 85,2 & 946,2 & 12860 & 42 & 73,7 & 326,6 \\
\hline Total & 72802 & 189 & 100,0 & 259,6 & 61914 & 57 & 100,0 & 92,1 \\
\hline
\end{tabular}


FIGURA 1. Incidencia de fractura de fémur proximal según grupo de edad y sexo. Mar del Plata, Argentina, 1992-1993

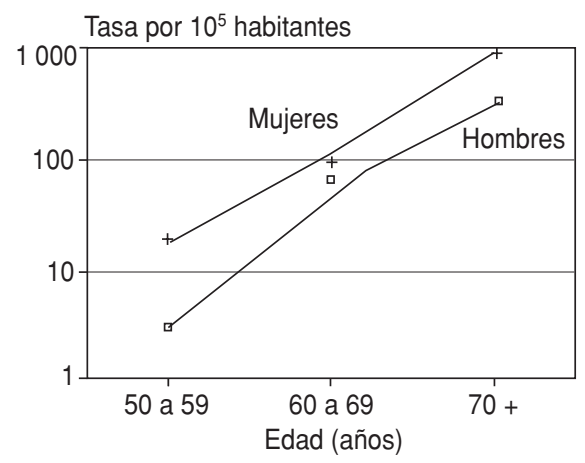

Fuente: Centros asistenciales de Mar del Plata, Argentina.

controles, mientras que los valores menores de $140 \mathrm{mmHg}$ se registraron en $15 \%$ de los casos y $25 \%$ de los controles, relación que produjo una razón de oportunidades 2,25 veces mayor en los casos. En la presión arterial diastólica no se encontraron diferencias estadísticamente significativas, pues $66 \%$ de los casos y $73 \%$ de los controles tuvieron valores menores de $90 \mathrm{mmHg}$. Tampoco se observaron diferencias significativas en la frecuencia cardíaca.

Con respecto a afecciones oculares, $44,4,38,9$ y $5,6 \%$ de los casos presentaron problemas visuales, cataratas y glaucoma, respectivamente, frente a 47,41 y $6 \%$ de los controles. Las diferencias también fueron mínimas en cuanto a salud de los pies y de la dentadura y el empleo de prótesis: $27 \%$ de los casos y $39 \%$ de los controles tenían problemas de los pies; $45 \%$ de los casos y $49,5 \%$ de los controles carecían de dientes propios y $37,8 \%$ de los casos y $49,5 \%$ de los controles empleaban prótesis dentales, totales o parciales.

En las mujeres, la edad promedio de inicio de la menopausia fue 48,28 años $( \pm 5,07)$ en los casos y 47,54 años $( \pm 5,27)$ en los controles. En 45,9\% de los casos y $47,7 \%$ de los controles, la menopausia se inició pasados los 50 años de edad. Nunca tuvieron hijos $17,6 \%$ de los casos contra $13,1 \%$ de los controles, mientras que $17,4 \%$ y $26,7 \%$ respectivamente, tuvieron más de cuatro hijos.
Solamente $7 \%$ de los casos y $6 \%$ de los controles habían sufrido ooforectomías.

En el momento de la entrevista, 23\% de los casos y $11 \%$ de los controles refirieron consumir alcohol $(P=0,04)$. En cuanto a cantidad semanal, $18 \%$ de los casos y $10 \%$ de los controles ingerían entre dos y siete vasos a la semana, en tanto que $3 \%$ de los casos y $0,9 \%$ de los controles tomaban ocho vasos o más. Entre los casos, 5,6\% informaron que fumaban frente a $9,3 \%$ de los controles. De los fumadores, $34 \%$ de los casos y $60 \%$ de los controles consumían más de 20 cigarrillos al día.

En lo que se refiere a la ingesta de productos lácteos, los consumían $82,4 \%$ de los casos y $84 \%$ de los controles. La ingesta era de más de tres vasos de leche al día en $7 \%$ de los casos y $18 \%$ de los controles. No hubo diferencias significativas en relación con la exposición a la luz solar; $34 \%$ de los casos y $40 \%$ de los controles indicaron que no tomaban sol.

Los pacientes con fracturas habían tenido un mayor número de fracturas previas $-34,2 \%$ de los casos contra $19,6 \%$ de los controles-, lo que significó un riesgo relativo 2,13 veces mayor para los primeros. En relación con enfermedades concomitantes, el porcentaje de enfermedades neurológicas fue más elevado entre los casos $(35,2 \%)$ que entre los controles $(16,8 \%)$ (razón de posibilidades $=2,68$ ). También fue más alto en los casos el porcentaje de enfermedades cardíacas $(45,4 \%)$ que en los controles $(38,3 \%)$ (razón de posibilidades $=1,34$ ). Los porcentajes de diabetes, $13 \%$ en los casos y $10,3 \%$ en los controles, superaron el promedio correspondiente a la población en general.

Por último, el análisis del uso de medicamentos reveló que el consumo de psicofármacos, suplementos de calcio y tiazidas fue mayor en los casos que en los controles, con RP respectivas de 1,$48 ; 2,10$ y 2,04 (cuadros 2 y 3).

\section{DISCUSIÓN Y CONCLUSIONES}

La osteoporosis se considera una enfermedad multifactorial que se manifiesta en un aumento de la fragilidad ósea. Si bien es cierto que la pérdida de material óseo y el riesgo de caídas aumentan con la edad, también es verdad que su incidencia varía significativamente de una población a otra. Así, la fractura de cadera es menos frecuente en mujeres negras que en blancas y dentro de cada raza hay un $50 \%$ de diferencia entre mujeres y varones. Hace algún tiempo, esas diferencias se solían explicar sobre la base de la masa ósea $(35,36)$. Sin embargo, los bantúes de Sudáfrica tienen una masa ósea menor que las mujeres blancas y un porcentaje inferior de fracturas (37). Lo mismo se ha observado en el Japón (38). En consecuencia, todavía no es posible explicar las diferencias étnicas.

Los datos de 1991 sobre la incidencia de fractura de fémur proximal en la ciudad de Mar del Plata se aproximan a resultados obtenidos en Siena (Italia) en 1991, donde la incidencia fue de 192 casos por 100000 en las mujeres y 108 por 100000 en los varones (razón de mujeres a varones de 2,8:1). Difieren, en cambio, con los datos obtenidos en Malmo (Suecia) en 1991, con una incidencia de 911 por 100000 en las mujeres y de 387 por 100000 en los varones, (razón de mujeres a varones de 2,5:1). En un trabajo previo sobre la ciudad de La Plata se obtuvieron valores similares a estos últimos (39).

En diversos trabajos se ha sugerido que la edad, el sexo, la talla, la raza, el peso corporal, la fuerza muscular y la historia familiar de osteoporosis son factores que influyen en el desarrollo de esta enfermedad. Hay otros factores que están en discusión, como la ingesta de calcio y de proteínas, el tabaquismo, la vida sedentaria y la edad de inicio de la menopausia (29). Al parecer, también son importantes en la incidencia de fractura de cadera el contenido de médula ósea, la acumulación de microfracturas en el hueso y la geometría local del hueso $(30,31)$.

En este trabajo no se encontraron diferencias entre casos y controles en cuanto a índices antropométricos como talla, peso corporal, IMC e ICC. Tampoco se observaron diferencias relacio- 
CUADRO 2. Resultados del análisis estadístico de los factores de riesgo de la fractura de fémur proximal. Mar del Plata, Argentina, 1992-1993

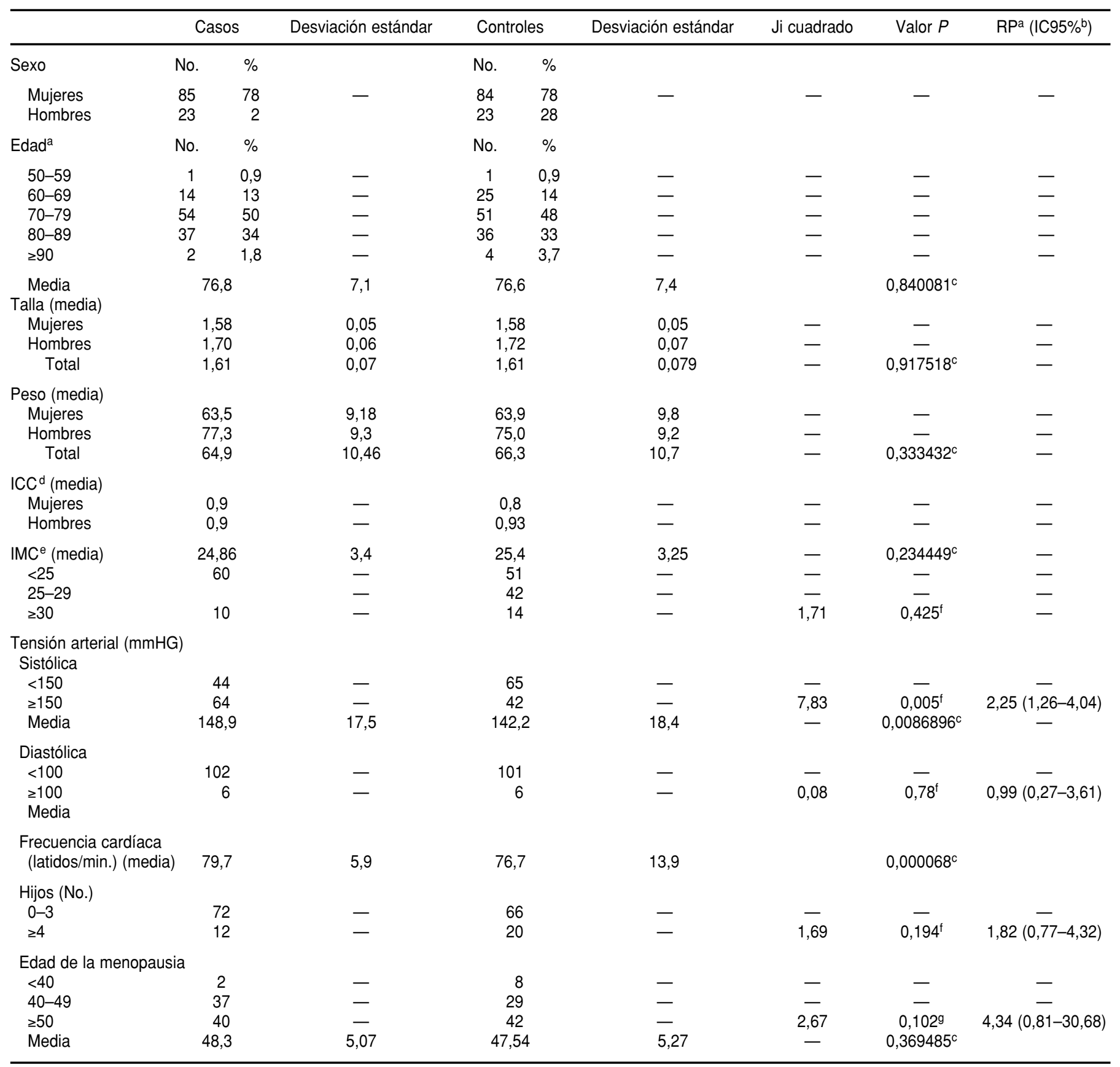

a $\mathrm{RP}=$ razón de posibilidades.

b IC95\% = intervalo de confianza de $95 \%$.

${ }^{c}$ Corresponde a la prueba de diferencia entre medias.

d ICC = índice cintura-cadera.

e IMC = índice de masa corporal.

${ }^{\dagger}$ Corresponde a la prueba de ji cuadrado.

${ }^{g}$ Corresponde a la prueba de ji cuadrado aplicada a los grupos $<40$ y $>40$ años, según criterio de los autores.

nadas con antecedentes de ooforectomía o con la edad de inicio de la menopausia. Antes de sufrir la fractura, $81 \%$ de los casos caminaban normalmente y sin asistencia. Otro hallazgo importante de este estudio fue el mayor porcentaje de fracturas previas entre los casos que entre los controles.
En Ginebra se observó que 21\% de los sujetos con fractura de cadera tenían antecedentes de fractura previa por osteoporosis. En otro estudio reali- 
CUADRO 3. Resultados del análisis estadístico de los factores de riesgo médicos, medicamentosos y nutricionales de la fractura de fémur proximal. Mar del Plata, 1992-1993

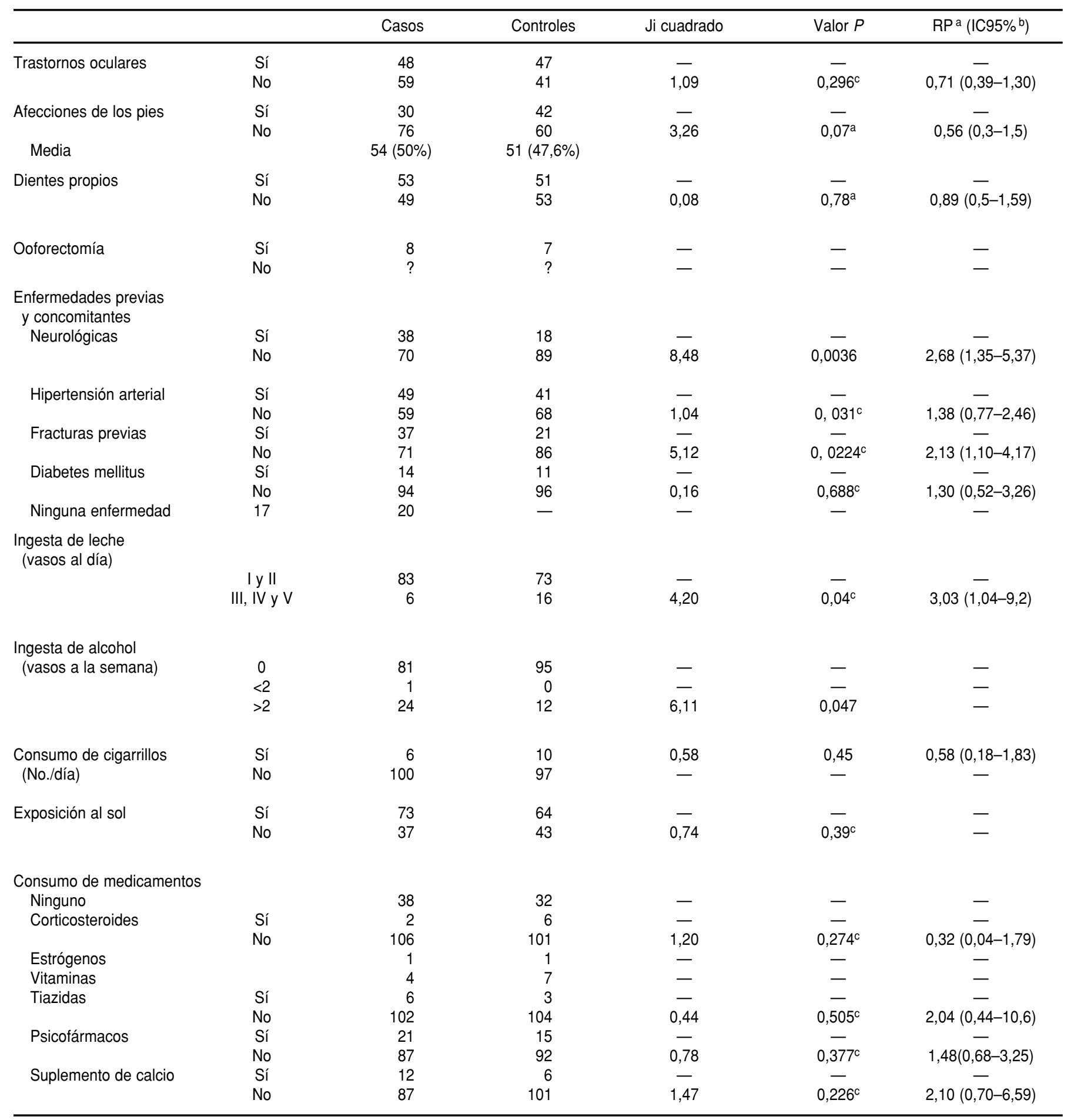

a RP = razón de posibilidades

b IC95\% = intervalo de confianza de 95\%.

c Corresponde a la prueba de ji cuadrado. 
zado en la Clínica Mayo (Estados Unidos), $68 \%$ de las mujeres y $59 \%$ de los hombres que habían sufrido fractura de fémur tenían como antecedente por lo menos una fractura anterior, la mayoría en columna vertebral o radio distal. Un hallazgo interesante fue que la fractura de cuello de fémur se asociaba con fractura previa en el radio distal o en la columna, pero raras veces con fractura previa de fémur $(40,41)$.

En lo respectivo a los factores extraóseos analizados en nuestro estudio, además de las caídas, entre los casos se observaron con mayor frecuencia que entre los controles estados patológicos subyacentes antes de la fractura. Hay indicios de que el exceso de mortalidad después de una fractura refleja la comorbilidad y fragilidad que la preceden, más que efectos de la fractura en sí, como lo han demostrado algunos trabajos en los que la osteoporosis sin fracturas también se asocia con un aumento de la mortalidad. En mujeres de edad avanzada con poca masa ósea, se han detectado aumentos importantes del riesgo de muerte sin fractura en los 3 años posteriores a un estudio (42). Otros trabajos muestran que los accidentes cerebrovasculares también constituyen factores de riesgo (43-45).

En el presente trabajo, los pacientes con fracturas mostraron mayor comorbilidad que los controles, hipertensión arterial sistólica más frecuente, mayor porcentaje de enfermedades neurológicas y mayor consumo de psicofármacos y de alcohol. No se encontró ninguna relación con la edad de inicio de la menopausia.
La expectativa mundial para reducir la incidencia de la fractura de fémur proximal se basa en la prevención (46-49). No obstante, los resultados inclinan a suponer que posiblemente la fractura de fémur proximal sea un marcador de los cambios que ocurren en nuestra sociedad. Esos cambios han llevado a la población a vivir más años, pero con estilos de vida que actualmente no implican desarrollar una estructura ósea y muscular lo suficientemente fuerte para prevenir las fracturas que hoy día son parte del envejecimiento. La vida moderna tiende, en general, hacia la inmovilidad física, sobre todo en el medio urbano. Varios estudios han revelado una relación inversa entre el porcentaje de fracturas y ciertos hábitos de vida, por ejemplo, con respecto a la ingesta de calcio (50) y a la actividad física (51). Hay muchas pruebas de que la actividad física regular reduce la pérdida ósea y, con ello, la frecuencia de fractura de fémur proximal (52-54). Quizá mediante una actividad física adecuada para personas de ambos sexos y el tratamiento hormonal en la mujer pueda reducirse en un futuro cercano la incidencia de fracturas óseas $(55,56)$.

Sobre la base de los datos anteriores, se concluye que en la población estudiada el riesgo de fractura aumentó a medida que aumentaba la edad de la población. La incidencia fue siempre mayor en las mujeres, con una razón de mujeres a hombres de 2,82:1. La incidencia, tanto específica por sexo como general, aumentó más rápida- mente en las mujeres de todos los grupos de edad. Los datos confirman observaciones anteriores de que la fractura proximal de fémur es un problema de especial importancia a partir de los 75 años de edad, tanto en hombres como en mujeres. En edades más tempranas, otras fracturas por osteoporosis son más importantes en términos de la carga de morbilidad y los costos de tratamiento médico.

Los datos también coinciden con trabajos anteriores en los que se determinó un mayor riesgo de fractura por osteoporosis después de sufrir una fractura traumática o atraumática. No se presentaron diferencias entre casos y controles en cuanto a peso, talla, IMC ni ICC. Con respecto a la posibilidad de establecer un índice de riesgo, por todo lo expuesto tendríamos que considerar que la masa ósea no es el único factor de riesgo de fractura. En nuestro estudio, los casos presentaron con mayor frecuencia que los controles diversos estados patológicos concomitantes, como enfermedades cardiovasculares y neurológicas, consumo de psicofármacos y alcohol y fracturas previas.

Agradecimiento. Los autores agradecen la colaboración de Lila Ricci, Universidad de Mar del Plata, María F. Aguirre, Instituto Nacional de Epidemiología Dr. Juan H. Jara, y María Patat, Universidad de Mar del Plata, en materia de informática y análisis estadísticos.

\section{REFERENCIAS}

1. Cooper C, Campion G, Melton LJ. Hip fractures in the elderly: a world-wide projection. Osteoporosis Int 1992;2:285-289.

2. Lindsay R. The burden of osteoporosis: cost. Am J Med 1995;98:9S-11S.

3. Schneider EL, Guralnik JM. The ageing of America. JAMA 1990;263:2335-2340.

4. Cummings SR, Rubin SM, Black D. The future of hip fractures in the United States: numbers, costs, and potential effects of post-menopausal estrogen. Clin Orthop 1990;52:163-166.

5. Fries JF, Koop CE, Beadle CE, Cooper PP, England MJ, Greaves RF, et al. Reducing health care costs by reducing the need and demand for medical services: The Health Project Consortium. N Engl J Med 1993;329:321-325.

6. Cooper C, Atkinson EJ, Jacobsen SJ, O'Fallon WM, Melton LJ. Population-based study of survival after osteoporotic fractures. Am J Epidemiol 1993;137:1001-1005.

7. Jensen JS, Bagger J. Long-term social prognosis after hip fractures. Acta Orthop Scand 1982;53:97-101.

8. Holbrook TL, Grazier K, Lelsey JL, Stanffer $\mathrm{RN}$. The frequency of occurrence, impact and cost of selected musculoskeletal conditions in the United States. Chicago: American Academy of Orthopedic Surgeons; 1984.
9. Zuckerman JD, Sakales SR, Fabian DR, Frankel VH. Hip fractures in geriatric patients. Clin Orthop 1992;274:213-225.

10. Phillips S, Fox N, Jacobs J, Wright WE. The direct medical costs of osteoporosis for American women aged 45 and older, 1986. Bone 1988;9:271-279.

11. Kellie SE, Brody JA. Sex-specific and racespecific hip fracture rates. Am J Public Health 1990;80:326-328.

12. Ray WA, Griffin MR, Baugh DK. Mortality following hip fracture before and after implementation of the prospective payment system. Arch Intern Med 1990;150:2109-2114. 
13. Chrischilles EA, Butler CD, Davis CS, Wallace RB. A model of lifetime osteoporosis impact. Arch Intern Med 1991;151:2026-2032.

14. Lu-yao GL, Baron JA, Barrett JD, Fischer ES. Treatment and survival among elderly Americans with hip fractures: a population-based study. Am J Public Health 1994;84:1287-1291.

15. White BL, Fisher WD, Laurin CA. Rate of mortality for elderly patients after fracture of the hip in the 1980s. J Bone Joint Surg Am 1987; 69:1335-1340.

16. Melton LJ III. Differing patterns of osteoporosis across the world. En: Chesnut CHI, ed. New dimensions in osteoporosis in the 1990s. Hong Kong: Excerpta Medica Asia; 1991. pp. 13-18.

17. Johnell O, Gullberg B, Allender E, Kanis JA. The apparent incidence of hip fracture in Europe: a study of national register sources. Osteoporosis Int 1992;2:298-302.

18. Cornell CN. Management of fractures in patients with osteoporosis. Orthop Clin North Am 1990;21:125-141.

19. Cummings SR, Kelsey JL, Nevitt MC, O'Dowd KJ. Epidemiology of osteoporosis and osteoporotic fractures Epidemiol Rev 1985; 7:178-208.

20. Garn SAM, Rohman CG, Wagner B. Bone loss as a general phenomenon in man. Fed Proc 1967;26:1729-1736.

21. Hui SL, Wiske PS, Norton JA, Johnston CC Jr. A prospective study of change in bone mass with age in post-menopausal women. $J$ Chronic Dis 1982;35:715-725.

22. Melton LJ III, Riggs BL. Epidemiology of agerelated fracture. En: de Aviolo LV. The osteoporotic syndrome. New York: Grune and Stratton; 1983. pp. 45-72.

23. Riggs BL, Melton LJ III. Involutional osteoporosis. New Engl J Med 1986;314:1676-1685.

24. Melton LJ III, Chrischilles EA, Cooper C, Lane AW, Riggs BL. How many women have osteoporosis? I Bone Miner Res 1992;7: 1005-1010.

25. Cummings SR, Black DM, Rubin SM. Lifetime risks of hip, Colles', or vertebral fracture and coronary heart disease among white postmenopausal women. Arch Intern Med 1989; 149:2445-2448.

26. Riggs BL, Wahner HW, Seeman E, Offord KP, Dunn WL, Mazess RB, et al. Changes in bone mineral density of the proximal femur and spine with aging: differences between the postmenopausal and senile osteoporosis syndromes. J Clin Invest 1982;70:716-723.

27. Bohr H, Schaadt O. Bone mineral content of femoral bone and the lumbar spine measured in women with fracture of the femoral neck by dual photon absorptiometry. Clin Orthop 1983;179:240-245.

28. Elsasser V, Hesp R, Klenerman L, et al. Deficit of trabecular and cortical bone in elderly women with fracture of the femoral neck. Clin Sci 1980;59:393-395.

29. Mazess RB. On ageing bone loss. Clin Orthop 1982;165:239-252.

30. Cummings SR. Are patients with hip fractures more osteoporotic? Am I Med 1985;78: 487-494.

31. Faulkner KG, Cummings SR, Black D Palermo L, Gluer CC, Genant HK. Simple measurement of femoral geometry predicts hip fracture: the study of osteoporotic fractures. J Bone Miner Res 1993;8:1211-1217.

32. Frankel VH. The ultimate strength of the femoral neck. En: Goff CW, ed. The femoral neck. Springfield, Illinois: C.C. Thomas Publishers; 1960 . pp. 48-58

33. Frankel VM, Pugh JW. Biomechanics of the hip. En: Tronzo RG, ed. Surgery of the hip joint. New York: Springer Verlag; 1984. pp. 115-131.

34. Nomura A, Wasnich RD, Heilbrun LK, Ross PD, Davis JW. Comparison of bone mineral content between Japan-born and US-born Japanese subjets in Hawaii. Bone Miner 1989 6:213-223.

35. Melton LJ III, Chao EYS, Lane J. Biomechanical aspects of fractures. En: Riggs BL, Melton LJ III, eds. Osteoporosis: etiology, diagnosis, and management. New York: Raven Press; 1988 pp. 111-131.

36. Kellie SE, Brody JA. Sex-specific and racespecific hip fracture rates. Am J Public Health 1990;80:326-328.

37. Solomon L. Bone density in ageing Caucasian and African populations. Lancet 1979;2: 1326-1330.

38. Ross PD, Norimatsu H, Davis JW, Yano K, Wasnich RD, Fujiwara S, et al. A comparison of hip fracture incidence among native Japanese-Americans and American Caucasians. Am J Epidemiol 1991;133:801-809.

39. Rubin Z, Bagur A, García M, Mautalen CA. Epidemiología de la osteoporosis en nuestro país. Rev Argent Geriatr Geront 1991;11:75-84.

40. Finsen V, Benum P. Past fractures indicate increased risk of hip fracture. Acta Orthop Scand 1986;57:337-339.

41. Nordin BEC, Peacock M, Aaron J, Marshall D, Crilly RG, Horsman A, et al. Osteoporosis and osteomalacia. Clin Endocrinol Metab 1980;9:177-205.

42. Browner WS, Seeley DG, Vogt TM, Cummings SR. Non-trauma mortality in elderly women with low bone mineral density. Lancet 1991;338:355-358.

43. Chiu KY, Pun WK, Luk KD, Chow SP. A prospective study on hip fractures in patients with previous cerebrovascular accidents. Injury 1992;23:297-299.

44. Finucane FF, Madans JH, Bush TL, Wolf $\mathrm{PH}$, Kleinman JC. Decreased risk of stroke among postmenopausal hormone users: results of a national cohort. Arch Intern Med 1993;153: 73-79.

45. Naessen T, Parker R, Persson I, Zack M, Adami HO. Time trends in incidence rates of first hip fracture in the Uppsala Health Care Region, Sweden, 1965-1983. Am I Epidemiol 1989;130:289-299.

46. Bennet NE. Hypertension in the elderly. Lancet 1994;344:447-449.

47. Brower WS, Seeley DG, Vogt TM, Cummings SR. Non trauma mortality in elderly women with low bone mineral density. Lancet 1994; 338:355-358.

48. Kreiger N, Kelsey JL, Holford TR, O'Connor T. An epidemiologic study of hip fracture in postmenopausal women. Am J Epidemiol 1982; 116:141-148.

49. Winner SJ, Morgan CA, Evans JG. Perimenopausal risk of falling and incidence of distal forearm fracture. $\mathrm{Br}$ Med J 1989;298: 1486-1488.

50. Lauritzen JB, Petersen MM, Lund B. Hip fractures prevented by external hip protectors: a randomised nursing home study. Acta Orthop Scand 1992;63(supl 248):84.

51. Riggs BL, Melton LJ III. The prevention and treatment of osteoporosis. N Engl J Med 1992; 327:620-627.

52. Weiss NS, Ure CL, Ballard JH, Williams AR, Daling JR. Decreased risk of fractures of the hip and lower forearm with post-menopausal use of estrogen. N Engl J Med 1980;303: 1195-1198.

53. Matkovic V, Kostial K, Smonovic I, Buzina R, Brodarec A, Nordin BEC. Bone status and fracture rates in two regions of Yugoslavia. Am J Clin Nutr 1979;32:540-549.

54. Kriska AM, Sandler RB, Cauley JA, LaPorte RE, Hom DL, Pambianco G. The assessment of historical physical activity and its relation to adult bone parameters. Am J Epidemiol 1988;127:1053-1063.

55. Astrom J, Ahnqvist S, Beertema J, Jonsson B. Physical activity in women sustaining fracture of the neck of the femur. J Bone Joint Surg [Br]1987;69:381-383.

56. Cooper C, Barker DJP, Wickman C. Physical activity muscle strength and calcium intake in fracture of the proximal femur in Britain. $\mathrm{Br}$ Med J 1988;297:1443-1446.

Manuscrito recibido el 3 de julio de 1996 y aceptado para publicación en versión revisada el 4 de abril de 1997. 
ABSTRACT Every year more than one million fractures of the proximal femur occur in the world, especially in older persons. Given the continuous aging experienced by populations, such fractures will become more frequent from year to year and will constitute a growing public health problem. The largest increase is expected to occur in countries of Latin America around the year 2050. Since nearly 70\% of all atraumatic fractures in persons over 45 are due to osteoporosis, a case-control study was conducted in the city of Mar del Plata, Argentina, for the purpose of investigating the incidence of and the risk factors associated with proximal femur fractures due to osteoporosis. Between 1 August 1992 and 31 July 1993, a record was kept of all fractures of the proximal femur due to osteoporosis in persons over 50 years of age that visited any of the city's 30 public and private health centers. A total of 246 cases was recorded. The incidence rate per 100000 inhabitants in the above-50 population was 259 among women and 92 among men, for a ratio of 2.8:1. The incidence was consistently higher in the older age groups, especially in persons over 75 . Factors associated with a statistically significant increased risk of fracture of the proximal femur were: a history of neurologic disorders, psychotherapeutic drug use, alcohol consumption, previous fractures, cardiovascular disease, and a decreased intake of milk products. There were no observed differences between cases and controls with respect to age at menopause, weight, height, previous activity, smoking habits, or sun exposure, nor were such differences detected in terms of the percentage of women who had undergone oophorectomy.

\section{Hospitales '98: Congreso Latinoamericano de Bioseguridad, Salud Ocupacional y Calidad Total en Hospitales y Laboratorios}

Fechas: 26 a 28 de noviembre de 1998

Lugar: Guayaquil, Ecuador

La Sociedad Ecuatoriana de Seguridad, Salud Ocupacional y Gestión Ambiental (SESO) y la Comisión Técnica Permanente de Riesgos Profesionales de la Organización Iberoamericana de Seguridad Social (OISS) anuncian este encuentro científico internacional dirigido a un amplio abanico de posibles participantes. El congreso será de interés para miembros de las disciplinas que se desempeñan en el medio hospitalario como las de salud y medicina, ingeniería sanitaria, trabajo social, psicología de hospitales, clínicas y laboratorios, seguridad relacionada con la salud ocupacional y el medio ambiente, administración de recursos humanos y control de la calidad, así como para miembros de comités de epidemiología y medicina preventiva, y de sindicatos y comités de empresas de hospitales y laboratorios. Entre los temas centrales proyectados figuran las auditorías de gestión de la salud ocupacional y la calidad en los centros hospitalarios, herramientas informáticas para la evaluación de riesgos en los hospitales, bioseguridad en los laboratorios, sida y hepatitis entre el personal sanitario, manejo seguro de desechos hospitalarios, planes de emergencia en hospitales, desastres y vulnerabilidad hospitalaria, control de radiaciones, estrés y salud mental en el personal.

Información:

Sociedad Ecuatoriana de Seguridad, Salud Ocupacional y Gestión Ambiental

Chambers 105 y Amarilis Fuentes (esquina)

Casilla 7015

Guayaquil, Ecuador

Teléfonos: (593-4) 330706 / 448 676; fax: (593-4) 580189 